\title{
Programa de Integración Escolar en Chile: brechas y desafíos para la implementación de un programa de educación inclusiva
}

\author{
School Integration Program in Chile: gaps and challenges for the \\ implementation of an inclusive education program
}

\begin{abstract}
Resumen
Construir sociedades inclusivas que no dejen a nadie atrás e incluyan a todos sus miembros es una obligación ética. Desarrollar programas de educación inclusiva permite asegurar igualdad de oportunidades en una de las etapas más críticas del desarrollo. El objetivo de este estudio es describir la implementación del Programa de Integración Escolar (PIE) en sus distintas dimensiones y zonas de Chile. Se desarrolló un estudio descriptivo y transversal midiendo la percepción de los coordinadores del programa en escuelas municipales y subvencionadas del país a través de una encuesta vía web. Se realizó un muestreo aleatorio simple de escuelas, obteniendo la respuesta de 1742 establecimientos educacionales con PIE. Se identificaron mayores niveles de implementación del programa en las áreas de trabajo interdisciplinar y formación integral, aspectos curriculares e institucionales. Por su parte, en la implementación de accesibilidad, desarrollo de ajustes razonables y participación de la comunidad educativa se identificaron deficiencias. Del mismo modo, existen diferencias entre las zonas de Chile, siendo la macrozona Norte la que presenta menor avance. Si bien hay resultados positivos en el equipo de trabajo y desarrollo institucional, el desarrollo de condiciones objetivas y la participación aún es una tarea pendiente en la implementación del PIE.
\end{abstract}

\section{Palabras clave}

Discapacidad, educación, escuela, inclusión, integración, necesidades educativas especiales, política educativa.

\footnotetext{
Abstract

Constructing inclusive societies, leaving no one behind, it is an ethical obligation. Developing inclusive educational programs allows ensuring equal opportunities in one of the most critical stages of development. The aim of this study is to describe the implementation of the School Integration Program (SIP) in its different dimensions and in different zones of Chile. A descriptive and cross-sectional study of the perception of SIP Coordinators was performed in public and subsidized schools at the country through a web-based survey. A simple random convenience sampling of schools was performed, obtaining 1742 answers from educational establishments with SIP. Higher level of implementation of the program was identified in areas related to interdisciplinary work and comprehensive training, curricular and institutional aspects. On the other hand, deficiencies were identified in the implementation of accessibility, development of reasonable adjustments and participation of the educational community. Likewise, there are differences between the zones of Chile, with the North zone having the least progress. Although there are results in the work team and institutional development, the development of objective conditions and participation is still a pending task in the implementation of the SIP.

\section{Keywords}

Disability, education, school, inclusion, integration, special educational needs, educational policy.
}

\author{
Mauro Tamayo Rozas \\ $<$ mtamayo@med.uchile.cl> \\ Universidad de Chile. Chile
}

\section{María Florencia Carvallo Arrau \\ <flocarvalloa@gmail.com> \\ Universidad de Chile. Chile}

\section{Magdalena Sánchez \\ Cornejo}

<mai.sanchezc@gmail.com>

Universidad de Chile. Chile

\section{Álvaro Besoaín-Saldaña <alvarobesoain@med.uchile.cl> Universidad de Chile. Chile}

Jame Rebolledo Sanhueza <jamerebolledo@med.uchile.cl>

Universidad de Chile. Chile

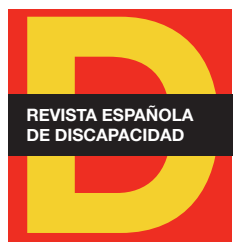

Para citar:

Tamayo, M. et al. (2018): "Programa de Integración Escolar en Chile: brechas y desafíos para la implementación de un programa de educación inclusiva". Revista Española de Discapacidad, 6 (I): 161-179.

Doi: <https://doi.org/10.5569/23405104.06.01.08>

Fecha de recepción: 13-01-2017 Fecha de aceptación: 12-12-2017 


\section{Introducción}

La educación inclusiva es un derecho humano fundamental para todos los estudiantes y permite la realización de otros derechos. Este derecho abarca una transformación en la cultura, la política y la práctica en todos los entornos educativos, sean estos formales o informales, para dar cabida a las diferentes necesidades e identidades de cada estudiante, junto con un compromiso para eliminar las barreras que impidan esa posibilidad. Además implica fortalecer la capacidad del sistema educativo para alcanzar a todos los estudiantes, centrándose en la participación plena y efectiva, accesibilidad, asistencia y logro de todos los estudiantes, especialmente de aquellos que, por diferentes razones, están en situación de exclusión o riesgo de marginalización.

La inclusión engloba el acceso y el progreso en una educación formal e informal de alta calidad, sin discriminación (Naciones Unidas, 2016). La educación inclusiva es considerada como la estrategia más eficaz para combatir las actitudes discriminatorias, guiada por la idea de que el sistema escolar debe adaptarse activamente a las condiciones individuales de aprendizaje de los niños y niñas, a fin de que puedan desarrollar todo su potencial. Desde un punto de vista sociológico, merece la pena destacar que la ambición de adaptar el sistema educacional a las necesidades de todos los niños está en consonancia con la comprensión del estrecho vínculo de la discapacidad con el medio social (Michailakis y Reich, 2009).

\subsection{Programa de Integración Escolar (PIE)}

En Chile existen claras diferencias en el acceso a la educación de personas en situación de discapacidad, en comparación con el resto de la sociedad. Por ejemplo, un 7,4 \% de los adultos en situación de discapacidad no recibieron ningún tipo de educación formal, mientras que en el resto de la población este porcentaje se limita al 1,3\%. Por otro lado, un 23,4\% presenta educación básica incompleta frente a un 11,3\% de las personas que no viven en situación de discapacidad (Senadis, 2015).

Los distintos esfuerzos en el desarrollo de programas que apuntan a la inclusión educativa buscan aumentar el impacto de políticas dirigidas a expandir las oportunidades de acceso y permanencia en la educación media y superior para los grupos más pobres, y enfrentar efectivamente la segmentación social de los contextos escolares y resultados de aprendizaje. Si bien, el análisis de la OCDE señala que - los programas de apoyo específico para las escuelas, aunque son efectivos, no son suficientes para reducir las desigualdades cuya naturaleza es más estructural y demandan un nuevo enfoque sistémico- (OCDE, 2004). Este desafío es aún mayor en términos de discapacidad, pues solo un 9,1\% de las personas en situación de discapacidad en Chile ha logrado la educación superior completa, frente a un $20 \%$ en el caso de personas sin discapacidad (Senadis, 2015). Estas diferencias siguen siendo injustas y evitables, por tanto, aún se requiere revisar constantemente la implementación de las estrategias de inclusión social.

Entre 1960 y 1970 surgieron en algunos países desarrollados críticas en contra de la forma segregada en que los niños con discapacidad recibían educación, y así la normalización y la integración fueron formuladas como objetivos de la política educativa (Michailakis y Reich, 2009). En la década de los setenta surge el concepto de necesidades educativas especiales (NEE), conceptualización que apunta a las características específicas que presenta un estudiante en cierto momento de su educación, las que no pueden ser aten- 
didas por los medios regulares necesitando mayores recursos para poder lograr el mismo aprendizaje que sus pares (Warnock Report, 1979).

En 1994 la Declaración de Salamanca de la UNESCO y el Marco de Acción sobre Necesidades Educativas Especiales proclaman la educación inclusiva como principio rector y afirman que: "Las personas con necesidades educativas especiales deben tener acceso a las escuelas ordinarias, que deberán integrarlos en una pedagogía centrada en el niño, capaz de satisfacer esas necesidades - (UNESCO, 1994). Dicho principio ha sido ratificado recientemente en el artículo 24 de la Convención sobre los Derechos de las Personas con Discapacidad (Naciones Unidas, 2006).

En Chile, en la década de los 90, nace el Programa de Integración Escolar (PIE) como una estrategia del sistema educacional que busca mejorar la calidad de la educación que se imparte en los establecimientos, favoreciendo la presencia y participación principalmente de los alumnos que presentan discapacidades, sean estas tanto de carácter transitorio como permanente, llamados estudiantes con NEE. Este programa, si bien tiene directa relación con la Ley 20.422 (Chile, 2010) que establece normas sobre igualdad de oportunidades e inclusión social de personas con discapacidad y constituye el marco jurídico más relevante para contribuir en la inclusión a nivel nacional, tiene carácter híbrido, pues convoca la lógica de los derechos humanos pero prescribe un modelo de integración con un fuerte énfasis psico-médico (López et al., 2014).

Para la implementación de este programa, se ponen a disposición del establecimiento recursos humanos y materiales adicionales, recursos asociados a los estudiantes ingresados en el programa, para así otorgar apoyo y entregar más oportunidades de aprendizaje y participación a los alumnos (Marfán et al. , 2013). Los estudiantes ingresados reciben un diagnóstico y un abordaje pedagógico individual con apoyo de un docente especialista. El establecimiento con implementación del programa debe contar con profesores regulares, profesores de educación diferencial, profesionales asistentes de la educación y fonoaudiólogos que contribuyan al proceso de aprendizaje del niño (Chile, 2009). A pesar de esto, un estudio cualitativo de López et al. (2014) señaló que esta política educativa, así como sus prácticas escolares y pedagógicas, al contrario de lo que pretenden, crean barreras para la inclusión en la medida en que tienden a la individualización, a la segregación y a la pérdida de la participación de los estudiantes con NEE.

\subsection{Derecho a la educación de las personas con discapacidad}

La Convención sobre los Derechos de las Personas con Discapacidad (CDPD) (Naciones Unidas, 2006) da cuenta de un profundo cambio de paradigma, donde la discapacidad es asumida como una cuestión de derechos humanos, relevando el papel que desempeña el entorno y la sociedad para facilitar o restringir la participación de las personas con discapacidad. Dentro de dichos obstáculos aparece, entre otras, la falta de voluntad política, conocimiento y capacidad, incluyendo al cuerpo docente para implementar la educación inclusiva, mecanismos de financiación inapropiados y/o normas o mecanismos legales para reclamar la reparación en situaciones en las que los derechos de las personas con discapacidad han sido vulnerados (Naciones Unidas, 2016).

Esta Convención llena un vacío en el marco de las normas internacionales de derechos humanos, detallando en sus artículos los derechos de las personas que viven en situación de discapacidad, que comprenden tanto 
los derechos civiles y políticos, como los derechos de accesibilidad, participación, inclusión, educación, salud, trabajo y la protección social, y supera la concepción de necesidades educativas especiales a personas con discapacidad (Naciones Unidas, 2006). Respecto a educación, señala en su artículo 24: - Los Estados Partes reconocen el derecho de las personas con discapacidad a la educación. Con miras a hacer efectivo este derecho sin discriminación y sobre la base de la igualdad de oportunidades, los Estados Partes asegurarán un sistema de educación inclusivo a todos los niveles, así como la enseñanza a lo largo de la vida-.

De esta manera, las exigencias y estándares de incorporar como eje de las políticas públicas la educación inclusiva, queda claramente expresada y se transforma en una obligación para los Estados. Crear escuelas inclusivas significa eliminar barreras para permitir el aprendizaje y la participación de todos los estudiantes. Esto implica la creación de culturas inclusivas, la elaboración de políticas inclusivas y el desarrollo de prácticas inclusivas (Booth y Ainscow, 2000).

\subsection{Aspectos clave de la inclusión escolar}

La inclusión educativa se entiende como un conjunto de procesos enfocados a eliminar o minimizar las barreras que limitan el aprendizaje y la participación del alumnado, particularmente en aquellos grupos de alumnos que podrían estar en riesgo de marginalización, exclusión o fracaso escolar (Echeita y Ainscow, 2011), por lo que se vuelve una herramienta crítica para el desarrollo de la educación en el marco de la CDPD.

En Chile y el mundo se han desarrollado diversos estudios para conocer los aspectos clave de la inclusión educativa, algunos de ellos indagando sobre la visión que tienen profesionales y alumnos en relación con la inclusión de estudiantes en situación de discapacidad. Entre sus resultados, se señala que "algunos de los factores que inciden en que no se dé una completa atención son: motivación de los docentes, exigencias del colegio y de los padres, falta de evaluación por parte de la institución al proyecto (...)" (Arias et al., 2005: 51). Existen diferentes miradas sobre sus resultados, por una parte, algunos autores defienden sus efectos positivos (Thomas, 1997; De Schauwer et al., 2009), otros enfatizan en las múltiples complejidades en su implementación (Clark et al., 1999; Norwich y Kelly, 2004) o la falta de evidencia sobre esta (Lindsay, 2007; Kelly et al., 2014).

Tanto las barreras como facilitadores de la inclusión se pueden encontrar en los elementos y estructuras del sistema -en escuelas, en la comunidad y en las políticas locales y nacionales- (Booth y Ainscow, 2000; Guerra et al., 2006). Para dar cumplimiento al acceso a una educación primaria y secundaria, de calidad y gratuita, el Comité de las Naciones Unidas sobre los Derechos de las Personas con Discapacidad (CRPD) señala que el sistema educativo debe comprender las cuatro siguientes características interrelacionadas: 1) disponibilidad de cupos, 2) accesibilidad, 3) aceptabilidad de la forma y fondo de la educación por parte de todos y 4) adaptabilidad del proceso de aprendizaje (Naciones Unidas, 2016). Por lo anterior, en un entorno basado en los principios de educación inclusiva, todas las personas de la comunidad escolar, independiente de su ascendencia, género, capacidad física o intelectual, religión, situación socioeconómica u otros factores similares, deben ser incluidos, respetados y tratados de manera justa. La diversidad se transforma en un valor que permite desarrollar una comunidad escolar segura, cómoda y aceptada (Ministerio de Educación de Ontario, 2010). 
La inclusión supone una nueva ética, unos nuevos valores basados en la igualdad de oportunidades. Los valores de la educación inclusiva tienen que ver con abrir la escuela a nuevas voces (las menos familiares) y con escucharlas activamente; pero también con el respeto y la redistribución de poder entre todos los miembros de la comunidad escolar. La nueva ética supone, en definitiva, pasar de aceptar la diferencia a aprender de ella (Parrilla, 2002).

En la práctica, la educación inclusiva requiere que el sistema educativo regular incorpore a los estudiantes con discapacidad en la misma clase que los estudiantes sin discapacidad (Gordon, 2013). A tales efectos la participación de los alumnos no debería consistir sólo en que los profesores y demás profesionales del centro valoraran a cada alumno y le reconocieran sus derechos, sino también en dotar a los alumnos de las herramientas necesarias para que ellos mismos puedan valorar su diversidad y conquistar sus derechos a ser educados y acogidos, para lo cual se requiere el compromiso de toda la comunidad escolar, y espacios y tiempos para reflexionar sobre las estrategias educativas que proporcionen un mayor valor al proceso de enseñanza-aprendizaje (Echeita y Sandoval, 2002).

Además del respeto a la diversidad y la participación de toda la comunidad escolar, un elemento material concreto para lograr la educación inclusiva es la accesibilidad, que debe incluir -los edificios, la comunicación e información, currículo, materiales educativos, métodos de enseñanza, evaluación y servicios de apoyo y lenguaje. El entorno de los estudiantes con discapacidad debe ser diseñado para mejorar la inclusión y garantizar su igualdad en toda su educación - (Naciones Unidas, 2016: 7). La accesibilidad universal - constituye un compromiso ético con las personas, teniendo en cuenta que vivimos en una sociedad pluralista en la que se producen demandas de justicia para atender a la diferencia. En tal contexto, es necesario contar con profesionales que desarrollen un ethos que favorezca un servicio profesional auténticamente universal, atento a la diversidad humana y que ofrezca diseños versátiles y ampliamente empoderantes, con el fin de atender al amplio espectro de necesidades humanas existentes - (Aparicio y Martínez, 2017: 39).

El trabajo coordinado entre docentes es también un elemento clave que asegura la adaptabilidad, el CRPD alienta a los Estados Partes para que apliquen el enfoque del Diseño Universal para el Aprendizaje (DUA), que permitiría el trabajo en entornos de aprendizaje adaptables para cubrir las necesidades diversas de todos los estudiantes (Naciones Unidas, 2016). Sin embargo, en las experiencias estudiadas por López et al. (2014), los estudiantes del PIE se entienden como responsabilidad exclusiva del profesor diferencial, y desde la perspectiva de los profesores las posibilidades de aprendizaje dependen de la discapacidad del estudiante y su apoyo familiar, restando importancia a la función pedagógica.

El presente artículo tiene como objetivo describir la implementación del Programa de Integración Escolar (PIE) entre sus distintas dimensiones y en distintas zonas de Chile a través de la percepción de coordinadores del programa en escuelas municipales y subvencionadas del país. 


\section{Método}

Este estudio es de tipo observacional, descriptivo y transversal. Esta encuesta de carácter auto-aplicada, fue resuelta por los coordinadores del programa durante un plazo máximo de dos meses a partir de la fecha de envío.

\subsection{Muestra}

Según el directorio de establecimientos del MINEDUC 2013, el universo correspondió a 4851 establecimientos educacionales con implementación del PIE. El tamaño muestral, en base a un muestreo de tipo aleatorio simple con un $95 \%$ de confianza y un $5 \%$ de margen de error, fue de 875 establecimientos educacionales con PIE, según la estimación de tamaño muestral para tamaños poblacionales conocidos de Vivanco (2005). Se envió el cuestionario al total de coordinadores del PIE a nivel nacional ( $N=4851)$, se reenvió en dos ocasiones posteriores con un plazo de dos semanas cada una, finalmente se obtuvo un total de 1742 encuestas completas.

\subsection{Instrumento}

Para esta investigación se elaboró una encuesta de 69 preguntas. En este instrumento se consultó por datos sociodemográficos y sobre nueve áreas de análisis a partir de las propuestas de Marfán et al. (2013) con base en el decreto supremo 170 sobre Orientaciones Técnicas para PIE (Chile, 2009). Además se consideraron preguntas descritas en el Índice de Inclusión de la UNESCO y el CSIE (Booth y Ainscow, 2000) en torno a la participación de la comunidad escolar (dimensión A) y al desarrollo de prácticas inclusivas (dimensión C). Las áreas incluidas fueron: 1) Accesibilidad, espacios y mobiliarios, 2) Materiales Audiovisuales, 3) Formación integral, 4) Trabajo interdisciplinar, 5)Trabajo en aula, 6) Aspectos curriculares, 7) Promoción de participación de todos los estudiantes, 8) Participación de todos los actores educativos en el proceso de aprendizaje y 9) Aspectos institucionales. Cada área constituye una dimensión en particular de la cual se desprenden diversas variables. Las preguntas formuladas utilizaron como escala de medición selección múltiple, escala Likert de 5 puntos, dicotómicas (sí/no, municipal/particular-subvencionado y urbano/rural). Este cuestionario fue validado por un comité de expertos en metodología de investigación, construcción y planificación de cuestionarios, junto a expertos en educación inclusiva. Posteriormente, fue sometido a pilotaje. Se obtuvo un coeficiente de fiabilidad mediante alfa de Cronbach de 0,878 con valores por ítem entre 0,873 y 0,880. Estos valores están dentro del rango aceptable (Tavakol y Dennick, 2011). Dentro de estos, los ámbitos con mayor consistencia interna son los aspectos curriculares, de trabajo interdisciplinar y de participación de los estudiantes en situación de discapacidad y la comunidad educativa.

Finalmente, fue enviada a través de plataforma web en agosto de 2014 a los establecimientos educacionales con implementación del PIE dentro de Chile. 


\subsection{Procedimiento y análisis}

Con los resultados obtenidos, se construyó una base de datos en Excel para la tabulación de los datos recolectados. Se utilizó el alfa de Cronbach para evaluar la consistencia interna. Se realizó un primer análisis de frecuencia y medidas de tendencia central. Se utilizaron tablas de contingencia con el fin de dilucidar las diferencias entre las distintas variables seleccionadas. Para la presentación de resultados, se agruparon en cinco áreas que fueron analizadas por región, para evidenciar su distribución en el país. Esta base fue analizada posteriormente a través del programa SPSS 21.0 para Windows.

\section{Resultados}

Se obtuvieron 1742 encuestas válidas. En base a las variables sociodemográficas analizadas, encontramos que en promedio los establecimientos han implementado el Programa de Integración escolar (PIE) por ocho años. La distribución por región, macrozonas, área de ubicación y dependencia administrativa se muestra en la Tabla 1. Cabe destacar que sobre el $60 \%$ de las respuestas obtenidas corresponden a establecimientos de administración municipal.

Los profesionales que más se encuentran vinculados al Programa de Integración Escolar, para satisfacer las necesidades de los estudiantes en situación de discapacidad, en orden decreciente, son psicólogo 94,0\%, educador diferencial 93,7\%, fonoaudiólogo 78,0\% y psicopedagogo $66,1 \%$.

\subsection{Accesibilidad, ajustes razonables y aspectos curriculares del PIE}

Los aspectos de accesibilidad y ajustes razonables que se desarrollan a través del PIE en Chile se expresan en la Tabla 2. La implementación de apoyos y asistencia para los niños con discapacidad de origen sensorial está por debajo del $20 \%$. Particularmente los establecimientos no cuentan con material en sistema de lectura braille, audio libros o personal capacitado en lengua de señas (8\%). En general no se identifican diferencias significativas entre las macrozonas, a excepción de la disposición de material educativo para atender las necesidades de los estudiantes en situación de discapacidad $(p=0,021)$, presentando la macrozona del Desierto un nivel de implementación bajo al porcentaje nacional, y la macrozona Productiva y Exportadora sobre el nivel nacional.

En la Tabla 3 se aprecia el nivel de cumplimiento del trabajo interdisciplinar y formación integral por parte de los establecimientos en cada macrozona. En general, los coordinadores del PIE señalan que las actividades se realizan de manera interdisciplinaria, desarrollando trabajo conjunto entre docentes regulares y profesionales de apoyo (86\%). En relación al trabajo colaborativo en las distintas actividades pedagógicas, las reuniones de los profesionales del PIE y el desarrollo de una planificación y organización para la enseñanza entre los docentes y los profesionales del PIE, existen diferencias significativas en relación a la tendencia nacional, dado que la franja central metropolitana presenta menor cumplimiento respecto al nivel país, mientras que la macrozona productiva exportadora está sobre el promedio nacional. 
Finalmente, desde los aspectos curriculares del PIE, la mayoría de los establecimientos (sobre el $70 \%$ ) declara contar con adaptaciones y apoyos adicionales en los aspectos de evaluación y enseñanza aprendizaje para los estudiantes en situación de discapacidad (Tabla 4). Sólo en el tiempo adicional de apoyo que contempla el Programa de Integración Escolar no se observan diferencias significativas $(p>0,05)$. En general la macrozona del desierto presenta niveles de implementación curricular del PIE inferiores al nacional.

Tabla 1. Distribución de los establecimientos que participaron en el estudio por macrozona*, región, área y dependencia administrativa $(n=1742)$

\begin{tabular}{|c|c|c|}
\hline Ámbito & Frecuencia & $\%$ \\
\hline Área rural & 439 & 25,2 \\
\hline Área urbana & 1303 & 74,8 \\
\hline Tipo de establecimiento: Municipal & 1099 & 63,1 \\
\hline Tipo de establecimiento: Particular Subvencionado & 643 & 36,8 \\
\hline Macrozona 1: Del desierto & 253 & 14,5 \\
\hline I Región de Tarapacá & 51 & 2,9 \\
\hline XV Región de Arica y Parinacota & 24 & 1,4 \\
\hline II Región de Antofagasta & 36 & 2,1 \\
\hline III Región de Atacama & 19 & 1,1 \\
\hline IV Región de Coquimbo & 123 & 7,1 \\
\hline Macrozona 2: Faja central metropolitana & 610 & 35,0 \\
\hline V Región de Valparaíso & 157 & 9,0 \\
\hline XIII Región Metropolitana (RM) & 335 & 19,2 \\
\hline VI Región de O’Higgins & 118 & 6,8 \\
\hline Macrozona 3: Productiva exportadora & 624 & 35,8 \\
\hline VII Región del Maule & 162 & 9,3 \\
\hline VIII Región del Biobío & 312 & 17,9 \\
\hline IX Región de la Araucanía & 150 & 8,6 \\
\hline Macrozona 4: Los lagos y canales australes & 255 & 14,6 \\
\hline X Región de Los Lagos & 150 & 8,6 \\
\hline XIV Región de Los Ríos & 69 & 4,0 \\
\hline XI Región Aysén & 19 & 1,1 \\
\hline XII Región de Magallanes y la Antártica Chilena & 17 & 1,0 \\
\hline
\end{tabular}

* División en macrozonas basado en Federico Arenas Vásquez. Informe Final: Evaluación de la División Político Administrativa vigente, 29 de octubre 2007.

Fuente: elaboración propia. 


\subsection{Participación de la comunidad educativa}

En cuanto a los aspectos vinculados a la participación de la comunidad educativa en el marco del PIE, destaca la baja participación de estudiantes en sus espacios de toma de decisión como son los centros de estudiantes. En menos del $50 \%$ de los establecimientos se declara que son considerados en sus directivas y decisiones (Tabla 5). En cuanto a la participación de la familia y la comunidad extramural en la mitad de los establecimientos son considerados en el trabajo realizado. Llama la atención que menos del $50 \%$ de los establecimientos, en todas las macrozonas, declara que se realizan capacitaciones periódicas sobre estrategias que potencien la inclusión a todos los actores educativos (Tabla 6). Sólo se identifican diferencias significativas entre las macrozonas en el involucramiento de la familia en la planificación de los procesos educativos, siendo menor a nivel nacional $(97,1 \%)$ y en la macrozona del desierto $(93,7 \%)$, y mayor en la macrozona de los lagos y canales australes (99,2\%).

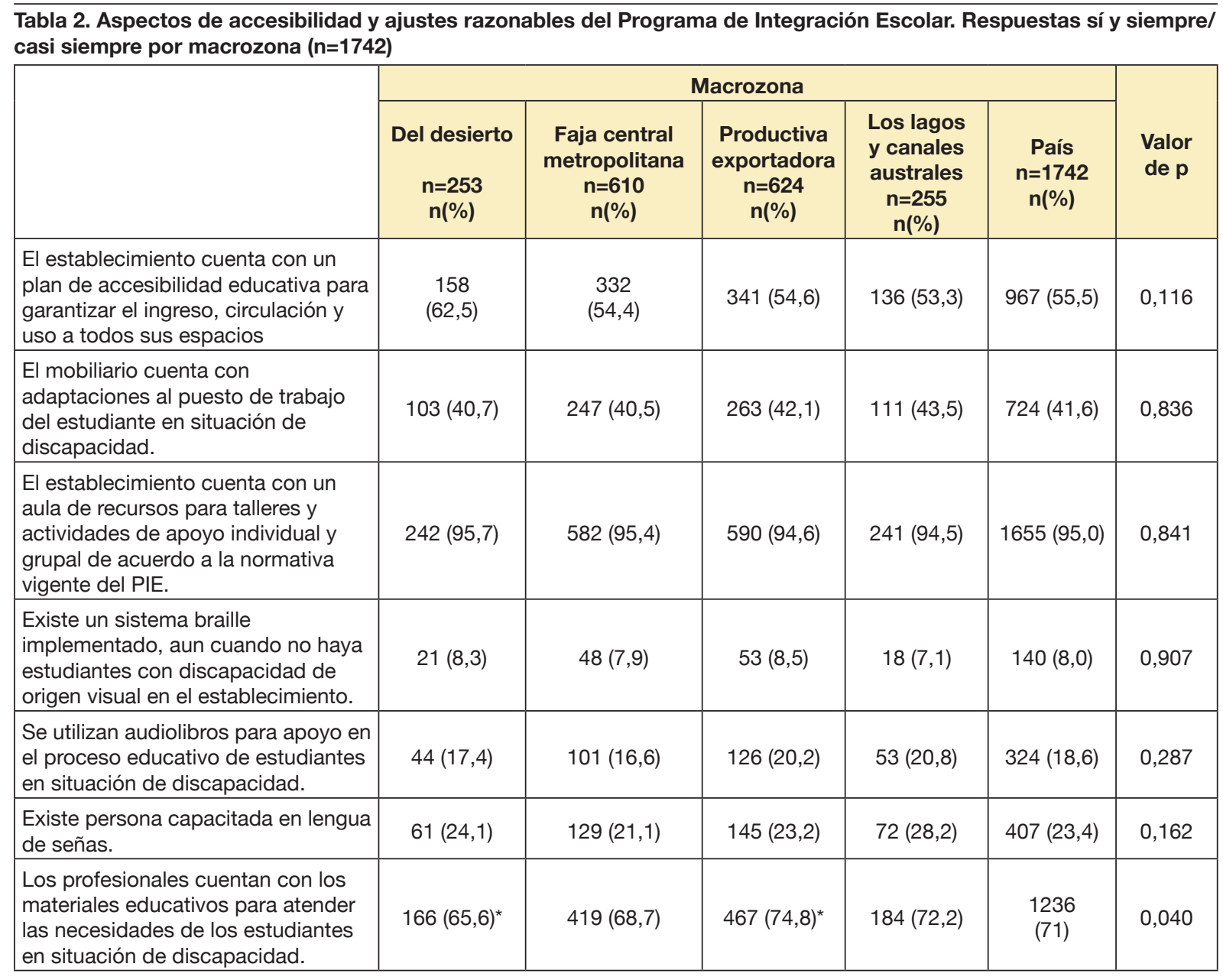

* Macrozonas con diferencias significativas $(p<0,05)$.

Fuente: elaboración propia. 


\section{Discusión}

Como principales hallazgos en las dimensiones de implementación del Programa de Integración Escolar (PIE) en Chile, encontramos mayores niveles de implementación de áreas de trabajo interdisciplinar y formación integral. Cerca del $90 \%$ de coordinadores del PIE señalan que las actividades se realizan de manera interdisciplinaria, mientras que desde los aspectos curriculares del PIE, más del $70 \%$ declara contar con

\begin{tabular}{|c|c|c|c|c|c|c|}
\hline & \multicolumn{5}{|c|}{$\begin{array}{c}\text { Macrozona } \\
\text { Recuento (\%) }\end{array}$} & \multirow[b]{2}{*}{$\begin{array}{l}\text { Valor } \\
\text { de } p\end{array}$} \\
\hline & $\begin{array}{c}\text { Del desierto } \\
n=253\end{array}$ & $\begin{array}{c}\text { Faja central } \\
\text { metropolitana } \\
n=610\end{array}$ & $\begin{array}{c}\text { Productiva } \\
\text { exportadora } \\
n=624\end{array}$ & $\begin{array}{c}\text { Los lagos } \\
\text { y canales } \\
\text { australes } \\
n=255\end{array}$ & $\begin{array}{c}\text { País } \\
n=1742\end{array}$ & \\
\hline $\begin{array}{l}\text { Se fomenta el trabajo } \\
\text { colaborativo en las distintas } \\
\text { actividades pedagógicas que } \\
\text { incluyen a los(as) estudiantes en } \\
\text { situación de discapacidad. }\end{array}$ & $214(84,6)^{\star}$ & $497(81,5)$ & $551(88,3)$ & $217(85,1)^{\star}$ & $1479(84,9)$ & 0,010 \\
\hline $\begin{array}{l}\text { Los profesionales del PIE } \\
\text { y docentes asisten a las } \\
\text { reuniones de planificación y } \\
\text { trabajan de manera colaborativa } \\
\text { para el diseño de las } \\
\text { planificaciones de enseñanza. }\end{array}$ & $210(83,0)^{\star}$ & $496(81,3)$ & $561(89,9)$ & $231(90,6)^{\star}$ & $1498(86,0)$ & 0,001 \\
\hline $\begin{array}{l}\text { Entre los docentes y los } \\
\text { profesionales del PIE existe, en } \\
\text { conjunto, una planificación y } \\
\text { organización para la enseñanza. }\end{array}$ & $203(80,2)^{\star}$ & $495(81,1)$ & $555(88,9)$ & $226(88,6)^{\star}$ & $1479(84,9)$ & 0,001 \\
\hline $\begin{array}{l}\text { Se toman en consideración } \\
\text { las recomendaciones de los } \\
\text { profesionales de apoyo. }\end{array}$ & $216(85,4)$ & $516(84,6)$ & $552(88,5)$ & $222(87,1)$ & $1506(86,5)$ & 0,232 \\
\hline $\begin{array}{l}\text { Las actividades realizadas } \\
\text { en el aula de recursos por } \\
\text { profesionales de apoyo son una } \\
\text { extensión de lo realizado en la } \\
\text { sala clases por los docentes. }\end{array}$ & $193(76,3)$ & $455(74,6)$ & $502(80,5)$ & $203(79,6)$ & $1353(79,7)$ & 0,074 \\
\hline $\begin{array}{l}\text { El (la) estudiante en situación } \\
\text { de discapacidad pasa la mayor } \\
\text { parte del tiempo posible en el } \\
\text { aula regular. }\end{array}$ & $241(95,3)$ & $575(94,3)$ & $581(93,1)$ & $236(92,5)$ & $1633(93,7)$ & 0,509 \\
\hline
\end{tabular}

* Macrozonas con diferencias significativas $(p<0,05)$.

Fuente: elaboración propia. 
adaptaciones y apoyos adicionales en los aspectos de evaluación y enseñanza aprendizaje para los estudiantes en situación de discapacidad. La incorporación del Diseño Universal del Aprendizaje (DUA) según lo estipula el Decreto Supremo 83/2015 (Chile, 2015), podría favorecer el trabajo interdisciplinar y las adaptaciones pedagógicas en las aulas de clase, esfuerzo concordante con lo señalado por Dalton et al. (2012) en el contexto de la implementación del DUA en Sudáfrica.

\section{Tabla 4. Aspectos curriculares del Programa de Integración Escolar. Respuestas sí y siempre/casi siempre por} macrozona $(n=1742)$

\begin{tabular}{|c|c|c|c|c|c|c|}
\hline & \multicolumn{5}{|c|}{ Macrozona } & \multirow[b]{2}{*}{$\begin{array}{l}\text { Valor } \\
\text { de } p\end{array}$} \\
\hline & $\begin{array}{l}\text { Del desierto } \\
\qquad n=253\end{array}$ & $\begin{array}{c}\text { Faja central } \\
\text { metropolitana } \\
n=610\end{array}$ & $\begin{array}{c}\text { Productiva } \\
\text { exportadora } \\
n=624\end{array}$ & $\begin{array}{c}\text { Los lagos } \\
\text { y canales } \\
\text { australes } \\
n=255\end{array}$ & $\begin{array}{c}\text { País } \\
n=1742\end{array}$ & \\
\hline $\begin{array}{l}\text { En el caso que un(a) estudiante } \\
\text { en situación de discapacidad } \\
\text { requiera tiempo adicional } \\
\text { de apoyo, el Programa } \\
\text { de Integración Escolar lo } \\
\text { contempla. }\end{array}$ & $201(79,4)$ & $470(77,0)$ & $484(77,6)$ & $202(79,2)$ & $1357(77,9)$ & 0,824 \\
\hline $\begin{array}{l}\text { Se realizan evaluaciones } \\
\text { que permiten reestructurar } \\
\text { los procesos de enseñanza } \\
\text { y aprendizaje de los(as) } \\
\text { estudiantes en situación de } \\
\text { discapacidad. }\end{array}$ & $199(78,7)^{\star}$ & $491(80,5)$ & $540(86,5)^{\star}$ & $218(85,5)$ & $1448(83,1)$ & 0,005 \\
\hline $\begin{array}{l}\text { El aprendizaje de los(as) } \\
\text { estudiantes se evalúa } \\
\text { adaptando el instrumento y } \\
\text { el nivel de dificultad utilizado } \\
\text { en base a las necesidades } \\
\text { que presente el estudiante en } \\
\text { situación de discapacidad. }\end{array}$ & $205(81,0)$ & $487(79,8)^{\star}$ & $557(89,3)^{*}$ & $221(86,7)$ & $1470(84,4)$ & 0,001 \\
\hline $\begin{array}{l}\text { Se proponen talleres y } \\
\text { actividades de apoyo individual } \\
\text { y grupal que refuercen el } \\
\text { aprendizaje para facilitar el } \\
\text { acceso al currículo }\end{array}$ & $193(76,3)^{\star}$ & $481(78,9)$ & $478(76,6)$ & $196(76,9)^{\star}$ & $1348(77,4)$ & 0,027 \\
\hline
\end{tabular}

* Diferencias significativas $(p<0,05)$.

Fuente: elaboración propia. 
Por otro lado, la implementación de accesibilidad y desarrollo de ajustes razonables es una de las áreas más débiles. Por ejemplo, la implementación de apoyos y asistencia para los niños con discapacidad de origen sensorial está por debajo del $20 \%$, especialmente herramientas como el sistema de lectura braille, audio libros o personal capacitado en lengua de señas (8\%). Esto implica que las escuelas en el periodo promedio de ocho años de implementación del PIE no han contado con recursos mínimos para el acceso inclusivo a libros o material de estudio. Esta situación puede ser atribuida a que el PIE incorpora mayoritariamente a "estudiantes con dificultades de aprendizaje" pues, según la normativa vigente (Chile, 2015), el personal predominante del PIE es psicólogo, quienes deben realizar la evaluación e identificación de las necesidades educativas especiales. Estas características del personal participante y los ajustes razonables presentes o, en su defecto, ausentes, dan cuenta de la poca preparación de las escuelas para el ingreso de estudiantes con discapacidad de origen sensorial o físico. Por lo anterior, es necesario identificar los diversos componentes necesarios para enfrentar los desafíos relativos a la inclusión escolar por medio de la evaluación de

\begin{tabular}{|c|c|c|c|c|c|c|}
\hline & \multicolumn{5}{|c|}{ Macrozona } & \multirow[b]{2}{*}{$\begin{array}{l}\text { Valor } \\
\text { de } p\end{array}$} \\
\hline & $\begin{array}{c}\text { Del } \\
\text { desierto } \\
\mathrm{n}=\mathbf{2 5 3}\end{array}$ & $\begin{array}{l}\text { Faja central } \\
\text { metropolitana } \\
n=610\end{array}$ & $\begin{array}{l}\text { Productiva } \\
\text { exportadora } \\
n=624\end{array}$ & $\begin{array}{l}\text { Los lagos } \\
\text { y canales } \\
\text { australes } \\
\mathrm{n}=255\end{array}$ & $\begin{array}{c}\text { País } \\
\text { n=1742 }\end{array}$ & \\
\hline $\begin{array}{l}\text { Las actividades están planificadas } \\
\text { para que participen todos los(as) } \\
\text { estudiantes sin restricción. }\end{array}$ & $222(87,7)$ & $554(90,8)$ & $575(92,1)$ & $234(91,8)$ & $1585(91,0)$ & 0,215 \\
\hline $\begin{array}{l}\text { Los(as) estudiantes en situación } \\
\text { de discapacidad participan sin } \\
\text { restricciones en el trabajo con el resto } \\
\text { de sus compañeros. }\end{array}$ & $226(89,3)$ & $562(92,1)$ & $577(92,5)$ & $231(90,6)$ & $1596(91,6)$ & 0,411 \\
\hline $\begin{array}{l}\text { Existen centros de alumnos que } \\
\text { incorporen a los estudiantes en } \\
\text { situación de discapacidad en sus } \\
\text { decisiones y directiva. }\end{array}$ & $124(49,0)$ & $231(37,9)$ & $297(47,6)$ & $106(41,6)$ & $758(43,5)^{\star}$ & 0,001 \\
\hline $\begin{array}{l}\text { Para planificar las actividades se } \\
\text { considera la opinión e interés de } \\
\text { los(as) estudiantes. }\end{array}$ & $178(70,4)$ & $409(67,0)$ & $451(72,3)$ & $172(67,5)$ & $1210(69,5)$ & 0,205 \\
\hline
\end{tabular}

* Diferencias significativas $(p<0,05)$.

Fuente: elaboración propia. 


\begin{tabular}{|c|c|c|c|c|c|c|}
\hline & \multicolumn{5}{|c|}{ Macrozona } & \multirow[b]{2}{*}{$\begin{array}{l}\text { Valor } \\
\text { de } p\end{array}$} \\
\hline & $\begin{array}{l}\text { Del desierto } \\
\qquad n=253\end{array}$ & $\begin{array}{l}\text { Faja central } \\
\text { metropolitana } \\
n=610\end{array}$ & $\begin{array}{c}\text { Productiva } \\
\text { exportadora } \\
n=624\end{array}$ & $\begin{array}{c}\text { Los lagos } \\
\text { y canales } \\
\text { australes } \\
n=255 \\
\end{array}$ & $\begin{array}{c}\text { País } \\
n=1742\end{array}$ & \\
\hline $\begin{array}{l}\text { Para planificar las actividades } \\
\text { se recoge información de la } \\
\text { comunidad. }\end{array}$ & $144(56,9)$ & $324(53,1)$ & $354(56,7)$ & $139(54,5)$ & $961(55,2)$ & 0,570 \\
\hline $\begin{array}{l}\text { Se toma en cuenta opinión de } \\
\text { la familia en la planificación de } \\
\text { los procesos educativos. }\end{array}$ & $156(61,7)$ & $335(54,9)$ & $429(68,8)$ & $173(67,8)$ & $1093(62,7)$ & 0,001 \\
\hline $\begin{array}{l}\text { Se realizan capacitaciones } \\
\text { periódicas sobre estrategias } \\
\text { que potencien la inclusión a } \\
\text { todos los actores educativos. }\end{array}$ & $108(42,7)$ & $276(45,2)$ & $305(48,9)$ & $102(40,0)$ & $791(45,4)$ & 0,078 \\
\hline $\begin{array}{l}\text { El trabajo realizado en la } \\
\text { escuela complementa al } \\
\text { trabajo realizado por la } \\
\text { familia y comunidad fuera del } \\
\text { establecimiento. }\end{array}$ & $129(51,0)$ & $322(52,8)$ & $366(58,7)$ & $145(56,9)$ & $962(55,2)$ & 0,088 \\
\hline
\end{tabular}

* Macrozonas con diferencias significativas $(p<0,05)$.

Fuente: elaboración propia.

áreas concretas (Guerra et al., 2006; Marfán et al., 2013), con tal de asegurar la efectividad de las estrategias de inclusión de niños y niñas en situación de discapacidad.

A su vez, el desarrollo profesional docente parece ser insuficiente en cuanto a la promoción de actividades de capacitación en inclusión social de los profesionales ejecutores del PIE. En este caso, se puede comprender que el desarrollo profesional docente no representa una fortaleza del PIE. Estos resultados concuerdan con los obtenidos por Arias et al. (2005), en un contexto local nacional, donde los profesores reconocen como desafíos - la capacitación de los profesores; mayor participación y compromiso de las familias; y por último un equipo profesional capacitado a los diferentes tiempos de aprendizaje de los niños con NEE-. En cuanto a los aspectos vinculados con la participación de la comunidad educativa en el marco del PIE, destaca la baja participación de estudiantes en sus espacios de toma de decisión como son los centros de estudiantes, y baja participación de la familia y comunidad extramural, lo que sería uno de los retos más importantes que deben afrontar los centros escolares, el relacionarse con las familias y con los diferentes miembros de la comunidad con la vista puesta en hacer de la escuela un proyecto compartido con mayores niveles de equidad (Simón et al., 2016). Esta condición es clave para la construcción de una sociedad de iguales que valore las diferencias.

Respecto al nivel de inclusión en términos generales, este programa recibe una evaluación favorable en la mayoría de los casos. Sin embargo, aquellas preguntas que buscan conocer aspectos más específicos y objetivos del establecimiento en sí, como accesibilidad y contar con ajustes razonables, revela la necesidad de mejoras sustantivas más allá de la voluntad de ser un establecimiento inclusivo. De hecho, el área con menor desarrollo es accesibilidad y diseño universal, ya que cerca de un $60 \%$ señala que el mobiliario 
no contaría con adaptaciones al puesto de trabajo del estudiante en situación de discapacidad. Esto es preocupante en la medida en que las dificultades más importantes en la experiencia que presentan los niños con discapacidad en la escuela, están en las instalaciones de aprendizaje, la empatía de sus pares y los obstáculos en el entorno físico para los estudiantes (Tran, 2014).

Llama especialmente la atención la presencia de diferencias significativas entre las macrozonas en: 1) Si se fomenta el trabajo colaborativo en las distintas actividades pedagógicas que incluyen a los estudiantes en situación de discapacidad; 2) Si los profesionales del PIE y docentes asisten a las reuniones de planificación y trabajan de manera colaborativa para el diseño de las planificaciones de enseñanza; 3) Si entre los docentes y profesionales del PIE existe, en conjunto, una planificación y organización para la enseñanza; 4) Si se toman en consideración las recomendaciones de los profesionales de apoyo. Estos elementos son centrales para el desarrollo de salas de clases inclusivas (UNESCO, 2016). Se requieren nuevos estudios a nivel regional que expliquen las diferencias encontradas a nivel de implementación de esta política de carácter nacional, ya que las diferencias de cobertura entre regiones y entre zonas urbano/rurales vistas en el informe de Marfán et al. (2013) son sustanciales.

Una limitación de este estudio es que la aplicación de la encuesta fue realizada sólo a los coordinadores del PIE. Aun cuando éstos son una parte importante de la comunidad escolar, representan una parte de la opinión de los actores del proceso educativo. Otra limitación de este estudio está relacionada con la técnica de recolección de datos, ya que este cuestionario no permite concluir el nivel de cumplimiento efectivo de las instituciones educativas con PIE, en relación con lo descrito tanto en las orientaciones técnicas como en las directrices planteadas por la UNESCO. Una aproximación observacional en las instituciones ayudaría a levantar información que no es capaz de ser recabada por los instrumentos, junto a variables que no son percibidas o relatadas por las personas.

Como proyección de este estudio se hace relevante incluir a los demás actores de la comunidad educativa para tener un panorama global y más completo de la realidad de la inclusión escolar en Chile. Esta profundización puede ser abarcada por medio del uso de la investigación cualitativa, particularmente metodologías participativas y etnográficas, que permitan recabar información de manera más intensa y válida sobre el proceso de inclusión escolar, información poco desarrollada en la literatura y absolutamente necesaria. Además, se considera como proyección para futuros estudios, estandarizar métodos de medición de la implementación de estos programas, con tal de desarrollar comparaciones sobre distintas regiones: América Latina, Europa, Asia, etc., que permitan generar procesos de cooperación en educación inclusiva con levantamiento de datos válidos y replicables.

La aplicabilidad de estos resultados al contexto educacional se puede analizar desde distintos ámbitos. En primer lugar, estos resultados son de utilidad para evaluar una correcta implementación del Programa de Integración Escolar tanto para entidades fiscalizadoras, supervisoras y ejecutoras tanto a nivel local como nacional, lo que puede apoyar un proceso de diagnóstico e implementación contextualizado de sus políticas públicas. Avanzar en la evaluación del impacto de las políticas de educación permitirá mejorar las estrategias de integración educativa y lograr la Inclusión en el aula y en la sociedad.

El presente estudio confirma lo señalado por Blanco y Duk (2011), que la mayoría de los países adoptan en sus leyes y políticas el derecho a la educación pero en la práctica es posible constatar que éste no se 
garantiza por igual a todas las personas y grupos sociales. Un sistema educativo justo no solo tiene que garantizar la igualdad en el acceso a los diferentes niveles educativos, sino también la democratización en el acceso al conocimiento y el respeto a las múltiples identidades de las personas, para que todos sientan que pertenecen a la sociedad y puedan participar en igualdad de condiciones.

Ampliar las oportunidades de todos para acceder a una educación de calidad y desarrollar escuelas más inclusivas, que eduquen en y para la diversidad, son dos poderosas herramientas para avanzar hacia sociedades más justas y democráticas en América Latina. Esta es una tarea de gran envergadura porque implica cambios profundos no solo en los sistemas educativos sino en el conjunto de la sociedad (Blanco y Duk, 2011).

Por lo anterior, podemos afirmar que la inclusión efectiva se logrará si modificamos nuestros paradigmas, si consideramos que la discapacidad no es un atributo inherente, es más bien un constructo social, ya que al hablar de discapacidad uno se está remitiendo a una categoría relacional, la cual se expresa en la interacción negativa entre el conjunto de barreras contextuales, actitudinales y ambientales con una persona en cualquier situación de salud, restringiendo el pleno goce de derechos y la autonomía de las personas (Tamayo, 2014). Con esta concepción sobre la discapacidad, el análisis en su dimensión educacional adquiere una dificultad mayor, puesto que la discapacidad se puede entender como una limitación impuesta al sujeto por el medio, tanto por las personas como por el espacio con el que se relaciona.

Es relevante tener en cuenta que, en distintos países, como Irlanda, un creciente número de estudiantes con discapacidad están abandonando las escuelas ordinarias y se están matriculando en las escuelas de educación especial. La principal razón que se esgrime está en el fracaso de las escuelas regulares para satisfacer sus necesidades de recursos académicos, sociales, emocionales, de comportamiento y de acceso a la salud. La experiencia de educación inclusiva en Irlanda es similar a la de los países que todavía luchan con la introducción de prácticas de inclusión en medio de restricciones socioeconómicas y educativas de recursos (Kelly et al., 2014), tal como se ha descrito en Chile.

\section{Conclusiones}

Esta investigación permitió generar información relevante sobre la implementación del PIE a partir de indicadores concretos y vinculados a los estándares de la educación inclusiva. Es posible afirmar que las áreas que requieren urgente trabajo y preocupación por parte de los actores vinculados al Programa de Integración Escolar (PIE) son, en primer lugar, la accesibilidad de instalaciones y materiales utilizados, seguido de la participación de los estudiantes en actividades curriculares y extracurriculares, y la capacitación y desarrollo profesional docente y de apoyo.

En el marco de establecer directrices para la necesaria reforma del sistema de educación para estudiantes en situación de discapacidad, se puede concluir que aún queda trabajo por realizar en el ámbito de la inclusión educacional, tanto en la entrega de condiciones objetivas que aseguren el desarrollo del potencial 
de los estudiantes con su entorno, como también en las condiciones subjetivas o sociales que den apoyo a la inclusión social. Este estudio aporta una mirada general a la implementación del PIE a nivel nacional, develando que son muchos los desafíos para alcanzar una educación inclusiva. 
Referencias bibliográficas

Aparicio, M. y Martínez Navarro, E. (2017): "Accesibilidad universal: sentido normativo e implicaciones en la educación y la práctica profesional”. Revista Española de Discapacidad, 5 (1): 25-41.

Arias, I. et al. (2005): Visión de la Integración de niños/as con NEE (Necesidades Educativas Especiales) desde la perspectiva de profesionales y alumnos/as (tesis de pregrado). Universidad de Chile, Santiago (en línea). <http://www.tesis.uchile.cl/tesis/uchile/2005/arias_i/sources/arias_i.pdf>, acceso 5 de noviembre de 2017.

Blanco, R. y Duk, C. (2011): “Educación inclusiva en América Latina y el Caribe”. Aula: Revista de Pedagogía de la Universidad de Salamanca, 17: 37-55.

Booth, T. y Ainscow, M. (2000): Índice de Inclusión. Desarrollando el Aprendizaje y la Participación en las escuelas. Inglaterra: Centre for Studies on Inclusive Education (CSIE).

Chile. Decreto 83 Exento, aprueba criterios y orientaciones de adecuación curricular para estudiantes con necesidades educativas especiales de educación parvularia y educación básica, Biblioteca del Congreso Nacional de Chile, 5 de febrero de 2015 (en línea). <https://www.leychile.cl/Navegar?idNorma=1074511>, acceso 26 de febrero de 2018.

Chile. Ley $\mathrm{N}^{\circ} 20.422$, establece normas sobre igualdad de oportunidades e inclusión social de personas con discapacidad, Biblioteca del Congreso Nacional de Chile, 10 de febrero de 2010 (en línea). <http://bcn.cl/1m09d>, acceso 23 de febrero de 2018.

Chile. Decreto Supremo $N^{\circ} 170 / 09$, fija normas para determinar los alumnos con necesidades educativas especiales que serán beneficiarios de las subvenciones para educación especial, Biblioteca del Congreso Nacional de Chile, 21 de abril de 2010 (en línea). <http://www.leychile.cl/Navegar?idNorma=1012570>, acceso 23 de enero de 2018.

Clark, C. et al. (1999): "Theories of Inclusion, Theories of Schools: deconstructing and reconstructing the 'inclusive school'”. British Educational Research Journal, 25 (2): 157-177.

Dalton, E. et al. (2012): "The implementation of inclusive education in South Africa: Reflections arising from a workshop for teachers and therapists to introduce Universal Design for Learning". African Journal of Disability, 1 (1): $1-7$.

De Schauwer, E. et al. (2009): “I Need Help on Mondays, It's Not My Day. The Other Days, I'm OK”. Perspectives of Disabled Children on Inclusive Education”. Children \& Society, 23 (2): 99-111.

Echeita, G. y Ainscow, M. (2011). "La educación inclusiva como derecho. Marco de referencia y pautas de acción para el desarrollo de una revolución pendiente". Il Congreso Iberoamericano sobre Síndrome de Down. Granada: Down España.

Echeita, G. y Sandoval, M. (2002): “Educación inclusiva o educación sin exclusiones”. Revista de Educación, 327 : 31-48 (en línea). <http://hdl.handle.net/10486/668119>, acceso 23 de febrero de 2018.

Gordon, J. S. (2013): “Is Inclusive Education a Human Right?”. The Journal of Law, Medicine \& Ethics, 41 (4): $754-$ 767.

Guerra, C. et al. (2006): Proyectos de integración escolar, factores que facilitan y obstaculizan su funcionamiento (tesis de pregrado). Universidad de Chile. Santiago (en línea) <http://www.tesis.uchile.cl/tesis/uchile/2006/ guerra_c/sources/guerra_c.pdf>, acceso 2 de noviembre de 2017. 
Kelly, A. et al. (2014): "Challenges in Implementing Inclusive Education in Ireland: Principal's Views of the Reasons Students Aged 12+ Are Seeking Enrollment to Special Schools". Journal of Policy and Practice in Intellectual Disabilities, 11 (1): 68-81.

Lindsay, G. (2007): "Educational psychology and the effectiveness of inclusive education/mainstreaming". British Journal of Educational Psychology, 77 (1):1-24.

López, V. et al. (2014): “Barreras culturales para la Inclusión: políticas y prácticas de Integración en Chile”. Revista de Educación, 363: 256-281.

Marfán, J. et al. (2013): Análisis de la implementación de los programas de integración escolar (PIE) en establecimientos que han incorporado estudiantes con necesidades educativas especiales transitorias (NEET) (en línea). <http://www.mineduc.cl/usuarios/edu.especial/doc/201402101719500.InformeEstudiolmplementacionPIE2013.pdf>, acceso 6 de noviembre de 2017.

Michailakis, D. y Reich, W. (2009): "Dilemmas of inclusive education”. ALTER, European Journal of Disability Research, 3 (1): 24-44.

Ministerio de Educación de Ontario (2010): The Ontario curriculum grades 1e8: Health and physical education, revised interim edition. Ottawa: The Queen's Printer.

Naciones Unidas (2016): CRPD/C/GC/4/2016, Comentario General N4. Artículo 24. Derecho a la Educación Inclusiva. Ginebra: ONU.

Naciones Unidas (2006): Convención de las Naciones Unidas sobre los Derechos de las Personas con Discapacidad (en línea). <http://www.un.org/esa/socdev/enable/documents/tccconvs.pdf>, acceso 15 de agosto de 2017.

Norwich, B. y Kelly, N. (2004): "Pupils' views on inclusion: moderate learning difficulties and bullying in mainstream and special schools". British Educational Research Journal, 30 (1): 43-65.

OCDE (2004): Revisión de Políticas Nacionales de Educación (en línea). <http://www7.uc.cl/webpuc/piloto/pdf/ informe_OECD.pdf $>$, acceso 15 de octubre de 2017.

Parrilla, A. (2002): "Acerca del sentido y origen de la educación inclusiva”. Revista de Educación, 327: 11-29.

Servicio Nacional de la Discapacidad (Senadis) (2015): // Estudio Nacional de la Discapacidad. ENDISC. Santiago de Chile: Ministerio de Desarrollo Social (en línea). <http://www.senadis.gob.cl/descarga/i/3959>, acceso 6 de noviembre de 2017.

Simón, C. et al. (2016): “Escuela, Familia y Comunidad: Construyendo Alianzas para Promover la Inclusión”. Revista Latinoamericana de Educación Inclusiva, 10 (1): 25-42.

Tamayo, M. (2014): "Presentación”, en Jeno, D. y Alburquerque, F. (eds.): Manual de fabricación de ayudas técnicas y asistencias tecnológicas a bajo costo. Santiago: Centro de Trastornos del Movimiento (CETRAM)- SENADIS (en línea): <http://cetram.org/wp/wp-content/uploads/2013/11/AT_manual_completo_2014.pdf>, acceso 22 de octubre de 2017.

Tavakol, M. y Dennick, R. (2011): "Making sense of Cronbach's alpha". International Journal of Medical Education, 2: 53-55.

Thomas, G. (1997): “Inclusive Schools for an inclusive society”. British journal of Special Education, 24 (3):103-107.

Tran, K. (2014): "Exploring the experience of children with disabilities at school settings in Vietnam context". SpringerPlus, 3 (103): 1-11. 
UNESCO (2016): Training Tools for Curriculum Development. Reaching out to all Learners: a Resource Pack Supporting Inclusive Education. Geneva: International Bureau of Education-UNESCO.

UNESCO (1994): Declaración de Salamanca de la UNESCO y el Marco de Acción sobre Necesidades Educativas Especiales. España: UNESCO (en línea). <http://www.unesco.org/education/pdf/SALAMA_S.PDF>, acceso 20 de septiembre DE 2017.

Vivanco, M. (2005): Muestreo Estadístico: Diseño y Aplicaciones. Santiago: Universitaria.

Warnock Report (1979): Committee of Enquiry into the Education of Handicapped Children and Young People. Special educational needs, report of Committee of Inquiry into Education of Handicapped Children and Young people. London: HMSO. 